\title{
An EMC study of an electrical powertrain for transportation systems
}

\author{
B. Chand ${ }^{1}$, K. Hähre ${ }^{2}$, J. Keghie ${ }^{1}$, and S. Dickmann ${ }^{1}$ \\ ${ }^{1}$ Fundamentals of Electrical Engineering, Helmut-Schmidt-University/University of the Federal Armed Forces, \\ Hamburg, Germany \\ ${ }^{2}$ Power Electronics, Helmut-Schmidt-University/University of the Federal Armed Forces, Hamburg, Germany \\ Correspondence to: B. Chand (benian.chand@hsu-hh.de)
}

\begin{abstract}
There are many reasons, why electric drive systems will be used in future more frequently in transportation systems. The electrification allows a flexible use of different energy sources and thus creates a greater independence from fossil energy. However, electric drive systems are a challenge for the manufacturers of transportation systems. The electrical powertrain can not only be exposed to interferences, it may also emit interferences into components and communication cables in the vicinity. This can be a high risk for the drive system and for the passengers. For that reason, an EMC study of an electrical powertrain for transportation systems is presented. There the interference at the unshielded high-voltage cable (HV-cable) is characterized. The analysis of the influence of position variation of the communication cable with respect to the unshielded HV-cables is one main aspect. Different optimizations have been added to the test setup.
\end{abstract}

\section{Introduction}

Almost every manufacturer of transportation systems extends his portfolio of products with electric drives. Not only a greater independence from fossil energy, but also more restrictive EMC-standards force the manufacturers to restructure their research and development departments. The electrical powertrain (Fig. 1) can not only be influenced from outside, it can also emit disturbances. These disturbances are critical, when important communication lines are influenced, which could put the drive or the safety of the passengers at risk. A simplified study was done in Weber et al. (2003). An extension to practical cases is done here. In addition, the interferences on the unshielded HV-cables are characterized. In transportation systems the HV-cables are distributed over the whole vehicle. Therefore the position between the HV-

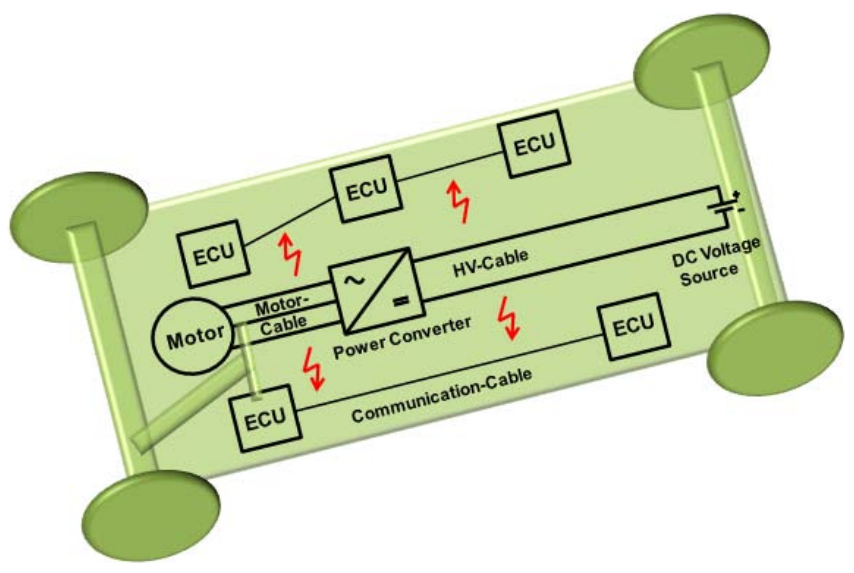

Fig. 1. Electrical powertrain and nearby Electronic Control Units (ECUs) in an automobile.

cables and existing communication lines can vary. And these different positions yields to different coupling behaviours.

For this reason, the position of the communication lines to the HV-cables is an essential point in this study. Furthermore, the influence of variations of some parameters is studied. These parameters which are taken into consideration are the motor speed, the PWM-frequency, the terminations and the load. An optimized control unit for the power converter is developed and built up in this study, and optimizations on the measurement setup are done. Different communication cables are used for comparison.

\section{Components of the test setup}

In Fig. 2 the measurement setup is shown. At first glance one could expect, that the disturbances are only present on the motor cables between the motor and the power converter. 


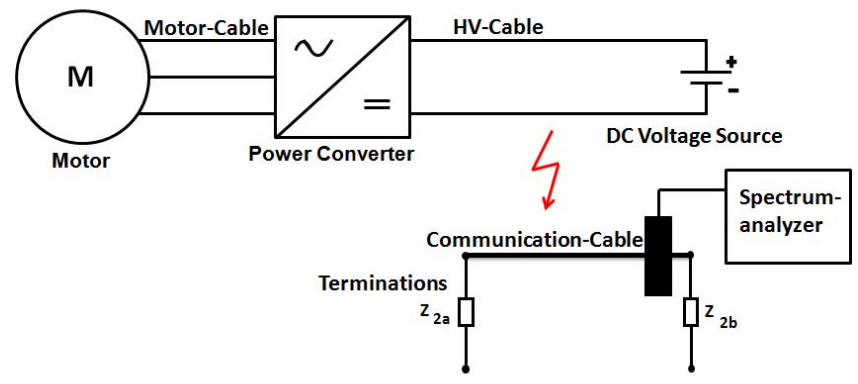

Fig. 2. Measurement setup.

This topic was presented in Kosonen and Ahola (2009). But this contribution shows, that disturbances are also present on the HV-cables at the DC side. Due to packaging reasons, the HV-cables are distributed over the whole vehicle and therefore could influence all components in the vehicle. The motor cables in Fig. 2 are kept very short or inserted into the housing of the motor, to minimize electromagnetic emissions.

\subsection{DC voltage source}

For the electrification of the powertrains, DC voltages from some hundred Volts to more than $1000 \mathrm{~V}$, depending on the power of the motor, are commonly used. In this study an adjustable source with maximum $350 \mathrm{~V}$ and $6.5 \mathrm{~A}$ is used.

\subsection{Power converter}

Power Converters generate from DC voltage/DC current a mono- or multiphase $\mathrm{AC}$ voltage/AC current with an adjustable frequency.

The frequency setting is done by a control unit. The schematic design of the inverter is given in Fig. 3 .

Two capacitors are used for smoothing and for buffering. The IGBT drivers control the six IGBTs, which are connected to form three half-bridges. At each of the transistors a freewheeling diode is attached, to protect the IGBTs from overvoltage. The inductors describe the motor in star circuit. The branch next to the two capacitors is the chopper. The chopper is used for recovery of the voltage, which is generated due to switching of the IGBTs. By matched time switching of the IGBTs an approximate three-phase sine wave is produced.

\subsection{Control unit}

A control unit for the inverter has been designed and built up in this study. It includes a 32-bit flash microcontroller, on the base of an ARM Cortex microcontroller architecture. With this control unit it is possible to create a three-phase PWM. Discontinuous step voltage profiles (e.g. rectangular patterns) cause electromagnetic interference, while by using the PWM method, a good approximation of a sine curve

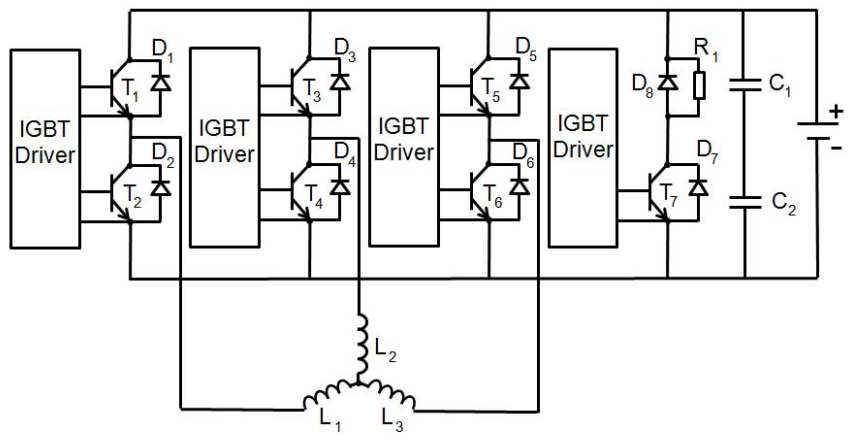

Fig. 3. Equivalent circuit of the power converter.

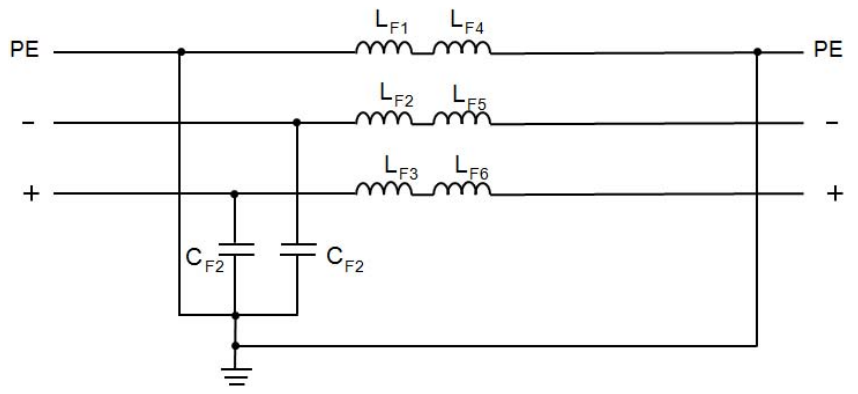

Fig. 4. Equivalent circuit of the DC-filter.

is created. Optocouplers are used for galvanic isolation of the signals. By using this control unit, it is possible to control the motor speed and the PWM-frequency. It should be noted, that the PWM frequency is chosen sufficiently large in comparison to the rotational frequency to obtain a good sine wave, and thus to ensure smooth running of the engine.

\subsection{Motor}

For the electrification of transportation means, direct current motors, asynchronous motors, synchronous motors and various coupled variants are used. In this study, a $5.5 \mathrm{~kW}$ asynchronous motor has been used, since induction motors are very robust and therefore widely used.

\subsection{Load}

Since the motor without a load only draws a small current, various loads can be used to allow considerable current flow. An elegant way to achieve this, are adjustable resistors, which are connected in parallel in a star circuit to the motor. Thus the current can be drawn from the system according to the values of the resistors.

\subsection{Cables}

Here unshielded HV-cables of $4 \mathrm{~mm}^{2}$ copper with a sheath of PVC have been used. For these studies single wire cables and twisted-pair cables as communication cables are used. The 


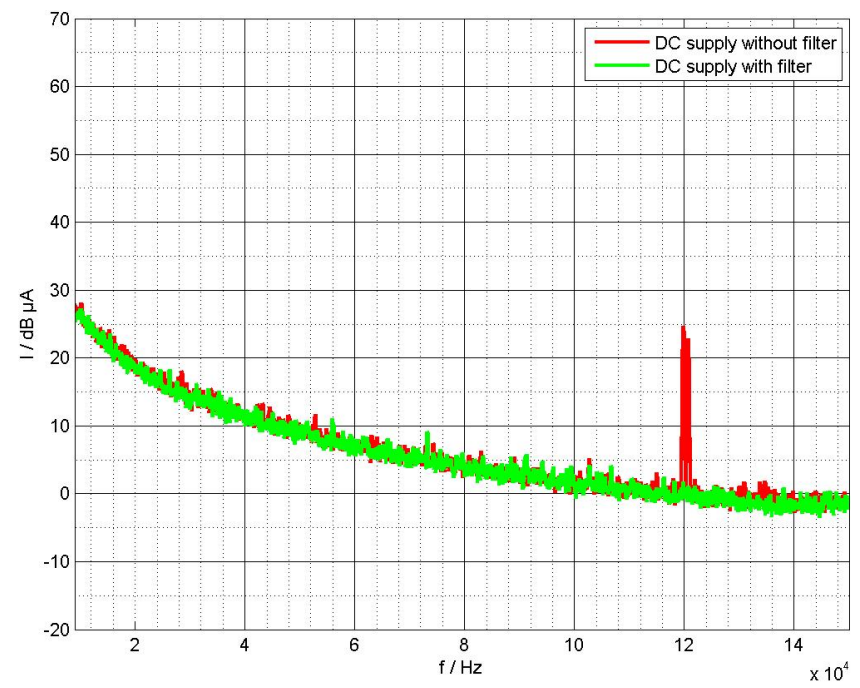

Fig. 5. Comparison of the results with filter and without filter, for the lower frequency range.

single wire is a copper wire with $0.75 \mathrm{~mm}^{2}$ cross section with a PVC jacket. The twisted-pair cable consists of two twisted wire strands, each $0.25 \mathrm{~mm}^{2}$, each with a PVC sheath. The single wire represents the communication line for the LINBus. While the bus line for the CAN-Bus and FlexRay-Bus is described by the twisted-pair cable.

\subsection{Spectrum analyzer}

The measurements are done using a signal analyzer with respect to IEC-CISPR16-2-1 (2008).

\section{Optimization of the test setup}

The measurements of the HV-cables show strong interferences, which come from the internal pulsed power supplies of the DC source. Since these unwanted interferences would distort the measurement results, a DC-filter (Fig. 4) was constructed and further optimized with simulations for the required frequency range.

The Figs. 5 and 6 show the results with and without filter for both frequency ranges. One can see a good attenuation of interferences from the pulsed DC voltage source. The filter improves the signal from the DC source significantly.

\section{Measurements and evaluation}

\subsection{Optimized measurement setup}

The interconnection of the components described in Sects. 2 and 3 yields the test setup shown in Fig. 7. The measurements are carried out in an Absorber Line Shielded Enclosure (ALSE) in order to avoid interferences from outside.

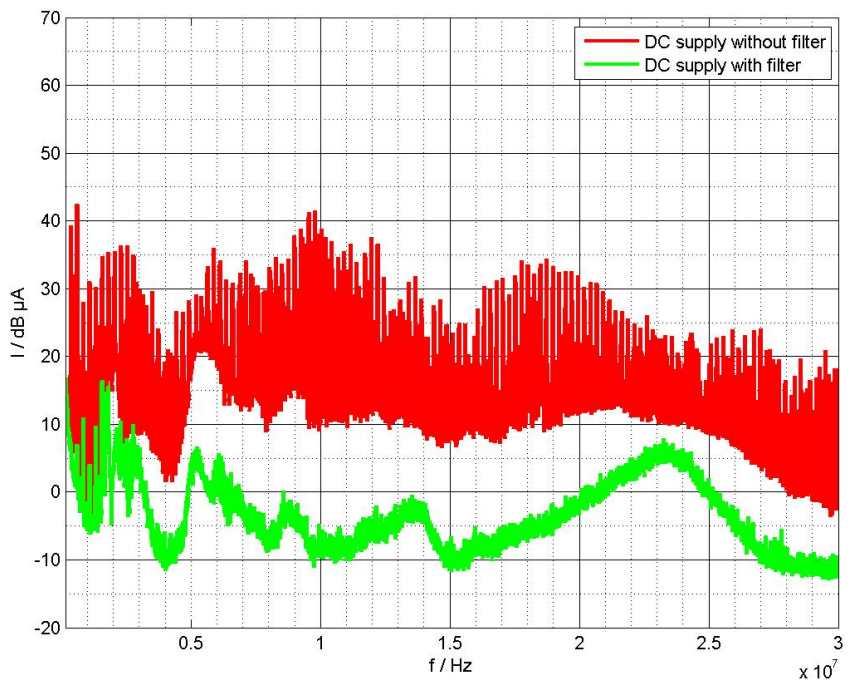

Fig. 6. Comparison of the results with filter and without filter, for the upper frequency range.

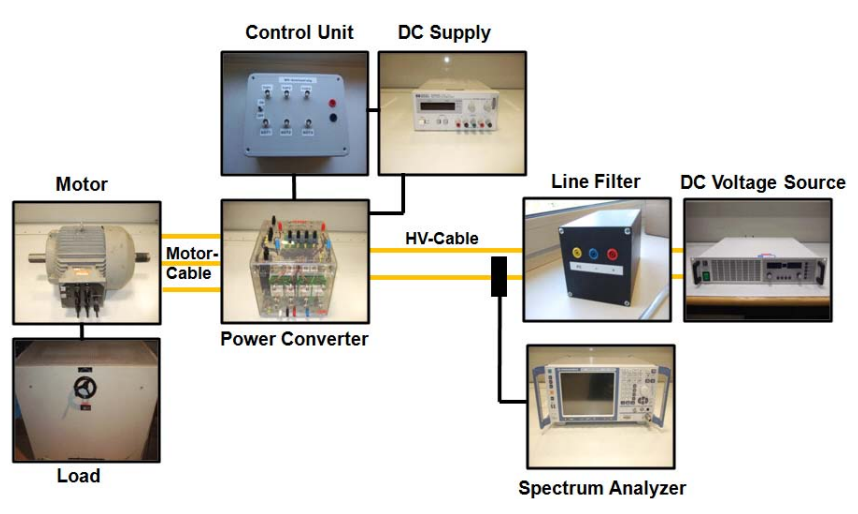

Fig. 7. Components of the whole test setup, connected to one possible measurement setup.

The spectrum has been measured with a current measuring clamp. Since the current through the cables is also of interest, one can calculate it using the frequency-dependent coupling factor $k$ of the clamp (Eq. 1).

$$
\frac{I}{\mathrm{~d} B \mu A}=\frac{U}{\mathrm{~d} B \mu V}+\frac{k}{\mathrm{~d} B \frac{1}{\Omega}}
$$

\subsection{Characterization of the HV-cable}

When measuring the current at various positions of both HV-cables, no significant differences in the results of the measurements are seen. On the motor cables, the PWMfrequency and its multiples can be seen clearly. In the higher frequency range the signal form and the magnitude change, like expected, when one compares the current of one cable to all three cables, due to the interaction of the currents on the three cables. The behaviour of the interferences on the 


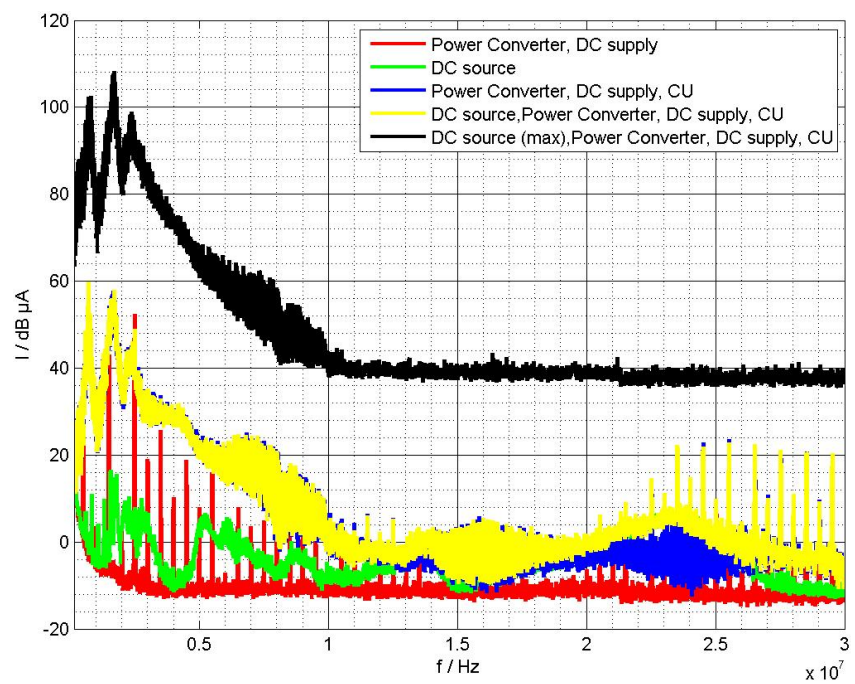

Fig. 8. Composition of the interference.

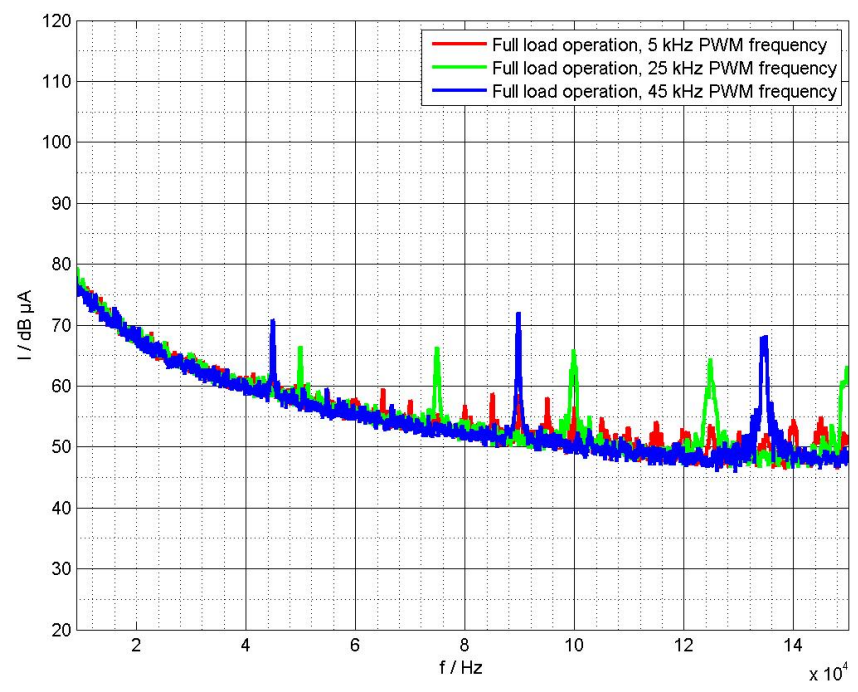

Fig. 9. Comparison of the results for different PWM-frequencies, for the lower frequency range.

HV-cables is composed of the frequency dependent internal interferences of the components, as illustrated by the following results in Fig. 8.

\subsection{Influence of the motor speed, the PWM-frequency and the load of the engine}

The change of the motor speed respectively the rotational frequency at constant PWM-frequency, does not change the interference behaviour. This is because the resulting shortterm disturbances are of transient nature and not considered here, since the frequency is measured under steady state condition. A change in the PWM-frequency at constant motor speed, however, shows a change of gradient. The higher the

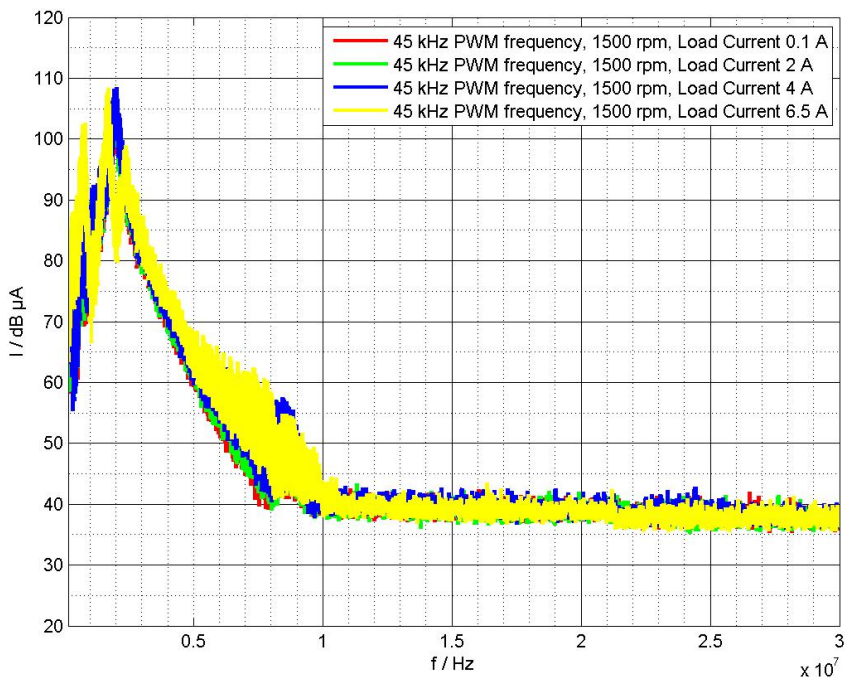

Fig. 10. Comparison of the results for different load currents, for the upper frequency range.

PWM-frequency, the higher the interference currents are and thus more resonances appear. In the lower frequency range, the PWM-frequencies and the multiples are clearly visible, (Fig. 9). Since higher PWM-frequencies lead to higher amplitudes, the PWM-frequencies should be kept as small as possible. But small PWM-frequencies lead to poor quality of the produced sine wave form. For these reasons a compromise between sufficiently high and minimum necessary PWM-frequency have to be found. An increase of the load, which results in an increase of the current, shows no significant differences in the frequency range of $9-150 \mathrm{kHz}$. In the upper frequency range of $150 \mathrm{kHz}-30 \mathrm{MHz}$, the magnitude changes of course at a higher current, but also the signal form of the interference. The signal form of the interference changes, because for different current/voltage ratio the conducted interference of the components changes internally, as shown in Fig. 10.

\subsection{Increasing the distance between the communication cable and the $\mathrm{HV}$-cables}

Increasing the distance between the measured cable and the $\mathrm{HV}$-cable in a parallel configuration yields as expected to decreasing magnitude.

In Fig. 11, three different values $(5 \mathrm{~cm}, 10 \mathrm{~cm}, 30 \mathrm{~cm})$ are used for the parallel distance between the cables. A decreasing of the parallel distance yields further to an increasing of the same ratio of $\mathrm{dB}$ of the interference current.

\subsection{Change of the height profile of the measured cable with respect to the reference surface}

Since the communication lines of transportation systems do not always have a fixed distance from the chassis, the 


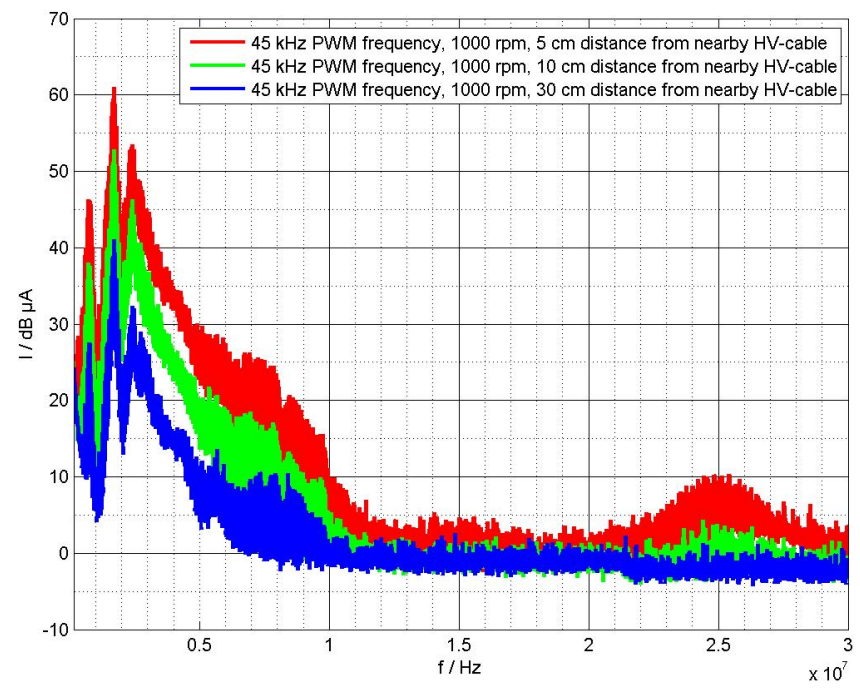

Fig. 11. Influence of position variation of the communication cable with respect to the HV-cables in a parallel configuration, for the upper frequency range.

influence on communication lines of different heights over a reference surface is studied. One side of the cable is set up $15 \mathrm{~cm}$ above the ground surface, the other is $5 \mathrm{~cm}$ above the ground plane. The result shows that such variation of the height profile of communication lines over a reference plane has a negligible influence on the interference behaviour for the studied case.

\subsection{Communication cable between the HV-cables, diagonally across the HV-cables and perpendicular to the HV-cables}

Furthermore, the influences of positioning the communication cable diagonally across the HV-cables, perpendicular to the HV-cables and between the HV-cables are compared. The results in Figs. 12 and 13 shows on the communication cable in the middle of the two HV-cables the most interferences. The inclined position of the measured cable causes a lower coupling of the interference current due to the coupling pattern of the E-and H-fields. The lowest coupling is thus obtained for vertically and centrally located communication cable. The change of the position of the communication cable yields up to $23 \mathrm{~dB}$ lower interferences.

\subsection{Influence of cable terminations at the communication cable}

Another important point is the investigation of the influence of the communication cable terminations. The terminations describe the input resistances in the ECUs in bus systems. The following Fig. 14 shows the results of the measurements with the different terminations.

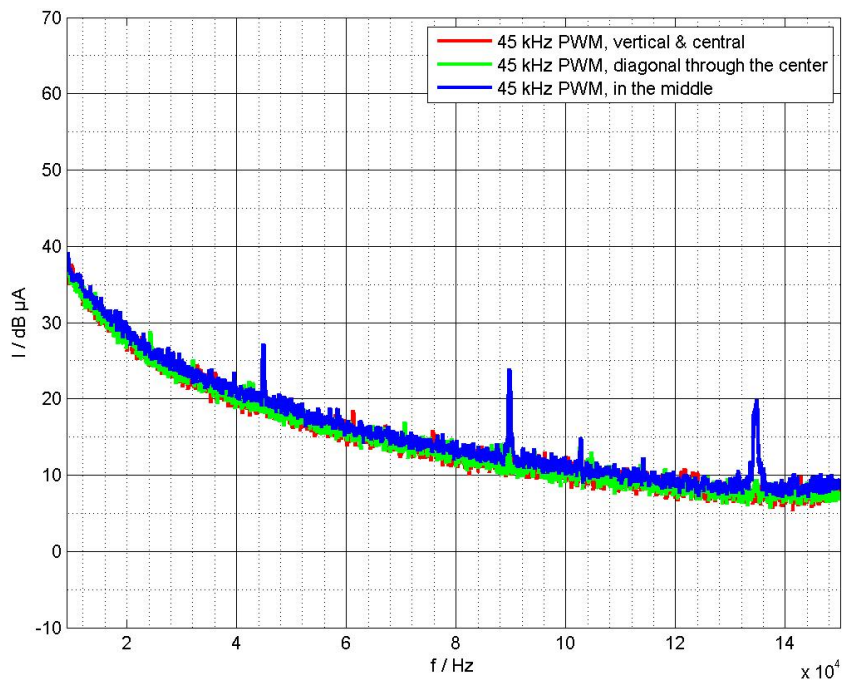

Fig. 12. Influence of position variation of the communication cable with respect to the HV-cables, for the lower frequency range.

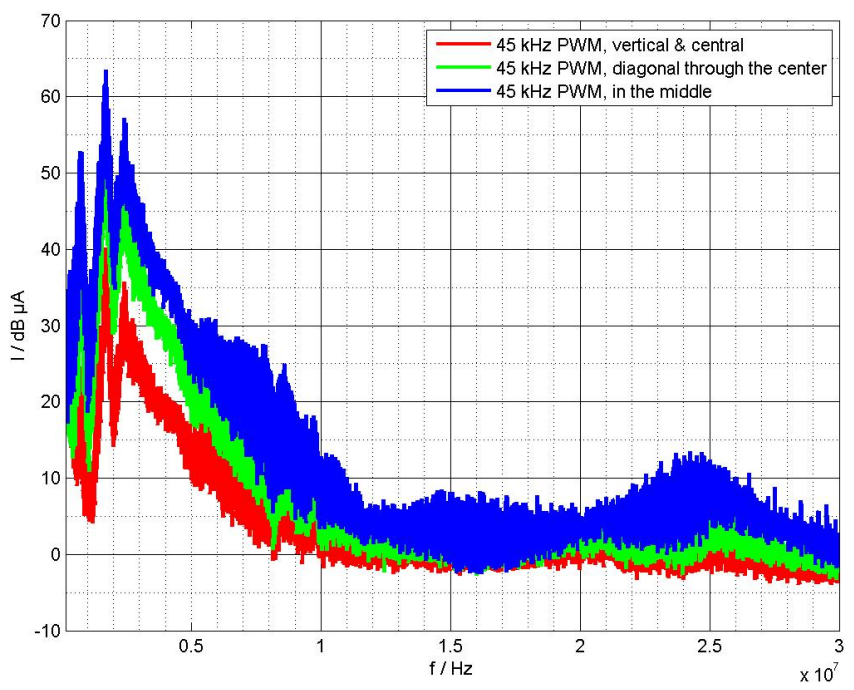

Fig. 13. Influence of position variation of the communication cable with respect to the HV-cables, for the upper frequency range.

It can be seen, that the terminations influence the standing wave patterns on the cables. There is a direct correlation between the choosen terminations and the current magnitude that can be measured. The differences can be up to $40 \mathrm{~dB}$, so it is necessary to consider the termination values well.

\subsection{Comparison of single wire and twisted-pair cable}

Not only the single-wire measurements are done, also the twisted-pair-cable, which represents the communication link for the CAN-Bus and FlexRay-Bus, is examined. This results for the low frequency range to no differences. For the B-Band (IEC-CISPR16-2-1, 2008) the current in the twistedpair-cable is twice as large than in the single wire, shown in 


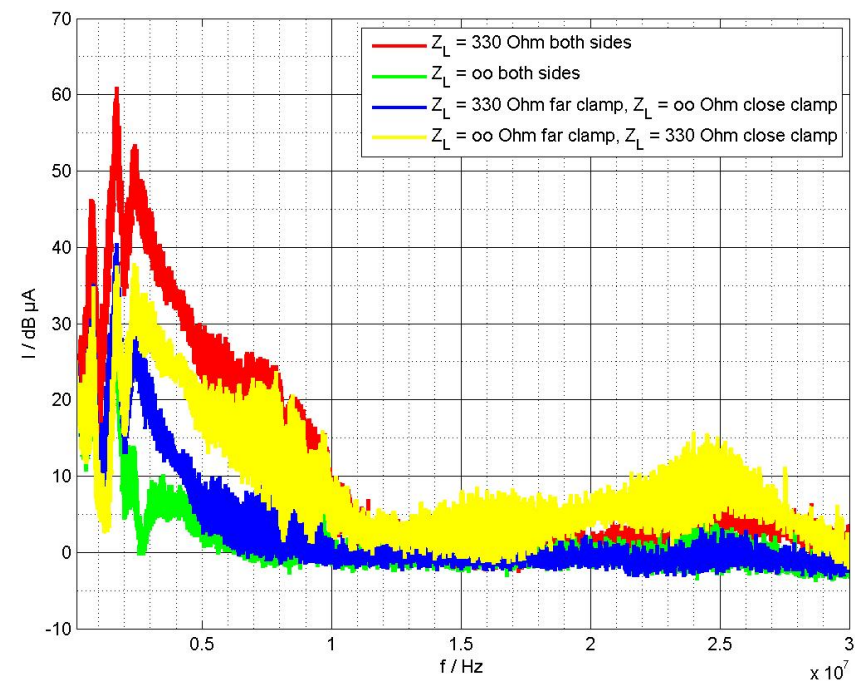

Fig. 14. Comparison of the results for different terminations for both ends of the communication cable, for the upper frequency range.

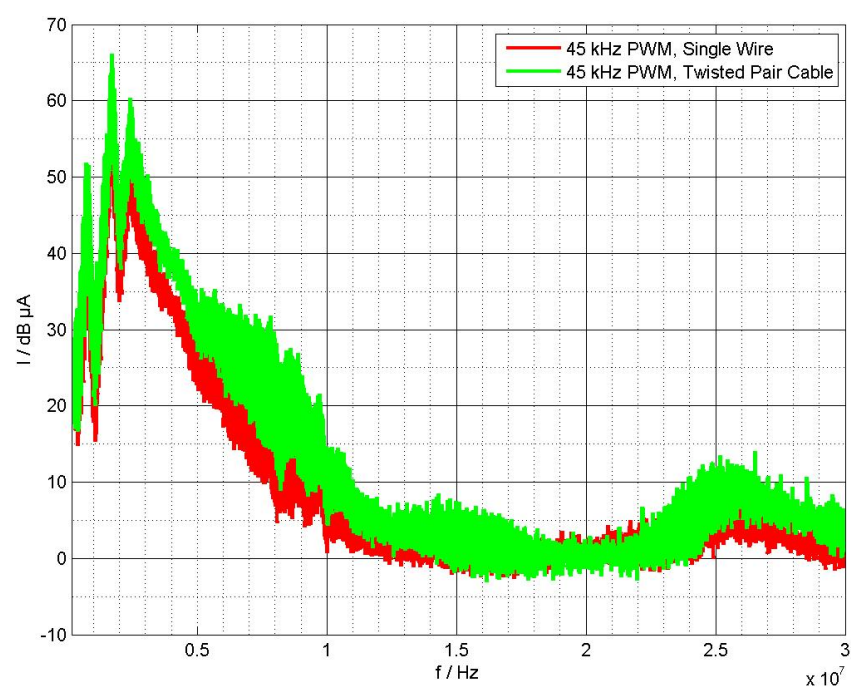

Fig. 15. Comparison of the results for single wire and twisted-pair cable as communication cable, for the B-Band.

Fig. 15. For that reason, as few wires as possible should be used. It should be noted, that the disturbances on the communication lines are common mode interferences.

The resulting currents can yield to load-voltages in Voltranges, depending on the terminations and on the voltage and current magnitude of the sources. For that reasons the interferences from the electrical drives to the communication systems should not be neglected, in order to avoid an influence up to a destruction of the communication.

\section{Conclusions}

In this paper an EMC study of an electrical powertrain for transportation systems is presented. The disturbance at the unshielded HV-cable is characterized. The analysis of the influence of position variation of the communication cable with respect to the unshielded HV-cables is studied. Furthermore, this contribution discussed the influence due to the variation of parameters like the motor speed, the PWM-frequency, the terminations and the load. An optimized control unit for the power converter is developed and built in this study, and optimizations at the test setup are done.

Due to different positions of the communication cables to the HV-cables the results were significantly improved.

To achieve a suppression of interferences on the communication lines, a meaningful positioning of communication cables with respect to HV-cables is encouraged. Using the knowledges gained in this contribution, the safety of the communication links can be increased.

\section{References}

IEC-CISPR16-2-1: Specification for radio disturbance and immunity measuring apparatus and methods Part 2-1: Methods of measurement of disturbances and immunity - Conducted disturbance measurements, 2008.

Kosonen, A. and Ahola, J.: Comparison of signal coupling methods for power line communication between a motor and an inverter, 2009, IET 2009, IET Electric Power Applications, 431-440, doi: 10.1049/iet-epa.2009.0114, 2009.

Weber, S., Guttowski, E., Hoene, E., John, W., and Reichl, H.: EMC Issues in Cars with Electric Drives, in: Electromagnetic Compatibility, 2003, EMC 2003, IEEE International Symposium on, 777-782, doi:10.1109/ISEMC.2003.1236706, 2003. 